\title{
Cortical Reorganization of Peripheral Vision Induced by Simulated Central Vision Loss
}

\author{
DNihong Chen, ${ }^{1,2}$ Kilho Shin, ${ }^{2}$ Rachel Millin, ${ }^{2,3}$ Yongqian Song, ${ }^{4}$ MiYoung Kwon, ${ }^{5}$ and Bosco S. Tjan ${ }^{2,3}{ }^{\dagger}$ \\ ${ }^{1}$ Department of Psychology, Tsinghua University, Beijing 100084, People's Republic of China, ${ }^{2}$ Department of Psychology, ${ }^{3}$ Neuroscience Graduate \\ Program, University of Southern California, Los Angeles, California 90089, ${ }^{4}$ School of Psychological and Cognitive Sciences and Beijing Key Laboratory of \\ Behavior and Mental Health, Peking University, Beijing 100871, People's Republic of China, and ${ }^{5}$ Department of Ophthalmology and Visual Sciences, \\ School of Medicine, University of Alabama at Birmingham, Birmingham, Alabama 35294
}

When one's central vision is deprived, a spared part of the peripheral retina acts as a pseudofovea for fixation. The neural mechanisms underlying this compensatory adjustment remain unclear. Here we report cortical reorganization induced by simulated central vision loss. Human subjects of both sexes learned to place the target at an eccentric retinal locus outside their blocked visual field for object tracking. Before and after training, we measured visual crowding-a bottleneck of object identification in peripheral vision, using psychophysics and fMRI. We found that training led to an axis-specific reduction of crowding. The change of the crowding effect was reflected in the change of BOLD signal, as a release of cortical suppression in multiple visual areas starting as early as V1. Our findings suggest that the adult visual system is capable of reshaping its oculomotor control and sensory coding to adapt to impoverished visual input.

Key words: central vision loss; cortical plasticity; crowding; fMRI; peripheral vision; PRL

\section{Significance Statement}

By simulating central vision loss in normally sighted adults, we found that oculomotor training not only induces PRL, but also facilitates form processing in peripheral vision. As subjects learned to place the target at an eccentric retinal locus, "visual crowding" - the detrimental effect of clutter on peripheral object identification-was reduced. The reduction of the crowding effect was accompanied by a release of response suppression in the visual cortex. These findings indicate that the adult visual system is capable of reshaping the peripheral vision to adapt to central vision loss.

\section{Introduction}

The human fovea typically serves as the locus for fixation because of its high visual acuity. When the fovea cannot provide detailed input-either due to retinal lesion or central vision deprivation, the oculomotor system sometimes adopts one or multiple locations in the peripheral retina for fixation, termed the preferred retinal locus (PRL; Cummings et al., 1985; Crossland et al., 2011; Kwon et al., 2013).

Received Aug. 21, 2018; revised Jan. 29, 2019; accepted Feb. 21, 2019.

Author contributions: N.C., M.K., R.M., and B.S.T. designed research; N.C., M.K., K.S., R.M., Y.S., and B.S.T. performed research; M.K. and B.S.T. contributed unpublished reagents/analytic tools; N.C., M.K., K.S., R.M., Y.S., and B.S.T. analyzed data; N.C. and M.K. wrote the paper.

This work was supported by National Institutes of Health (NIH)/National Eye Institute (NEI) Grants R01-EY017707 and P30-EY-029220. M.K. was supported by NIH/NEI Grant R01-EY-027857. We thank Gordon E. Legge and Daniel J. Kersten in the Department of Psychology at the University of Minnesota for helpful discussions. This article is dedicated to the memory of Bosco $S$. Tjan.

${ }^{\dagger}$ Deceased, December 2, 2016

The authors declare no competing financial interests.

Correspondence should be addressed to Nihong Chen at nihongch@usc.edu.

https://doi.org/10.1523/JNEUROSCI.2126-18.2019

Copyright $\odot 2019$ the authors
Nevertheless, object identification is impaired in the periphery. Crowding, a breakdown of target identification when surrounding objects are within a critical distance of the target (Bouma, 1970; Whitney and Levi, 2011), is considered as the primary limit for peripheral vision. Interestingly, a reorganization of the spatial extent of crowding has been reported in people with acquired central retinal lesion due to macular degeneration. While radially positioned flankers usually produce a stronger crowding effect than tangentially positioned flankers (Toet and Levi, 1992), this radial-tangential anisotropy was largely reduced in macular degeneration people at their PRL location (Chung, 2013).

To understand how developing a PRL reshapes the underlying visual processing, we simulated central vision loss in normally sighted adults with eye tracking. Subjects learned to use a peripheral retinal position for object tracking in a cluttered scene. Before and after training, we tested the spatial extent of crowding using psychophysics and fMRI. We found a radial-specific reduction of the crowding effect at the PRL location. The perceptual changes of crowding were associated with BOLD signal changes in multiple visual areas, starting as early as V1. 


\section{Materials and Methods}

\section{Subjects}

Twenty-eight healthy subjects ( 20 female; age range, 21-33 years old) participated in the experiment. They were naive to the purpose of the experiment and had never participated in any long-term training experiment before. One subject was excluded due to a technique issue with the eye tracking, and another was excluded due to excessive head movements in the scanner ( $>5 \mathrm{~mm}$ in a session). Nine subjects completed the main experiment for PRL training, nine completed the test-withouttraining experiment, and eight subjects completed the attention training experiment. All subjects had normal or corrected-to-normal vision. They had no known neurological or visual disorders. Informed consent was obtained in accordance with the procedures and protocols approved by the review committee of the University of Southern California.

\section{PRL Training}

Stimuli and apparatus. All stimuli were highcontrast 24 bit RGB color images of indoor scenes and objects. Images of indoor scenes and objects were selected from an image database (Luo et al., 2012) and from www. thinkstockphotos.com. The display was a 19inch CRT monitor (refresh rate, $85 \mathrm{~Hz}$; resolution, $1024 \times 768$ ). The stimuli were presented at a viewing distance of $57 \mathrm{~cm}$ with a subject's head stabilized by a chin and headrest. The displayed scenes subtended visual angles of $39^{\circ} \times 29^{\circ}$.

Eye movement recording and simulated scotoma. Subjects' eye movements were monitored with an infrared video-based eye tracker sampled at $2000 \mathrm{~Hz}$ (EyeLink 1000 Tower Mount Monocular Eye Tracker, SR Research) with a maximum spatial resolution of $0.02^{\circ}$. A gaze-contingent visual display was used to simulate central visual field loss in normally sighted subjects. The real-time gaze position was sent to the display computer through a high-speed Ethernet link. The average delay between eye movement and screen update was $\sim 10 \mathrm{~ms}$. Continuous gaze information was used to draw a scotoma on the display screen at a refresh and update rate of $85 \mathrm{~Hz}$. Given that a transient mismatch between the gaze and the scotoma location may occur when a subject either blinks or squints, our protocol minimized this mismatch by turning the entire display screen blank (gray) as soon as a blink was detected or the pupil size was decreased down to a threshold value (Aguilar and Castet, 2011). The scotoma was a circular gray patch (luminance, $18 \mathrm{~cd} / \mathrm{m}^{2}$ ) with a radius of $5^{\circ}$ (Fig. 1A). A gray cross at $6.5^{\circ}$ eccentricity, $30^{\circ}$ counterclockwise from the vertical meridian with respect to the fovea fixation serves as the gaze marker for the PRL. It was continuously presented to induce a PRL at the specified location.

Procedure. We simulated a central scotoma in normally sighted subjects to induce a PRL. Each trial started with a calibration and validation sequence followed by a drift correction. Subjects were instructed to follow a target object as it was randomly repositioned against a cluttered background (Fig. $1 B$ ). The center of the object was uniformly distributed within the central $31^{\circ} \times 22^{\circ}$ region of the display. Each target move was initiated after the PRL marker fell within the target region for $2 \mathrm{~s}$. After six repetitions, eight objects appeared simultaneously against the cluttered background. Subjects reported whether or not the target was present by a key press. The probability of the target being present was 0.5 . A correct response was followed by a tone. Each block consisted of 40 trials. Subjects completed $40-46$ blocks over 3-4 weeks. In a control experiment, the object-following task was replaced by a luminance change detection task to deploy subjects' attention at the PRL location. In each trial, a target object was presented at $6.5^{\circ}$ eccentricity, $30^{\circ}$ counterclockwise from the vertical meridian. The object changed its orientation every 100 $\mathrm{ms}$ and randomly dimmed during a $12 \mathrm{~s}$ presentation. Subjects were
B

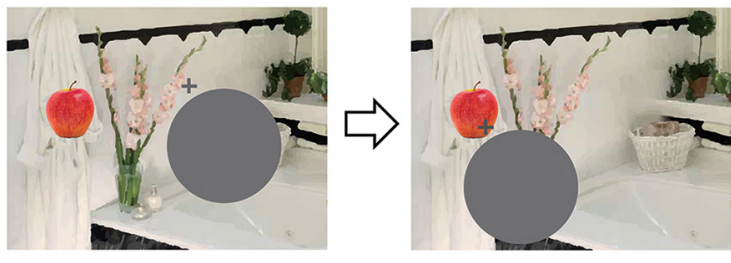

Start

Using PRL for target fixation

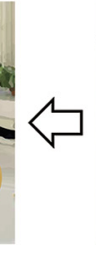

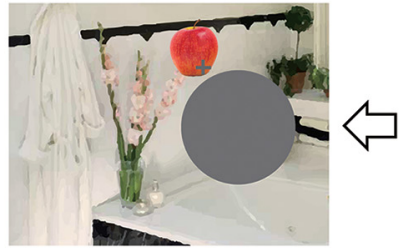

Using PRL for target fixation

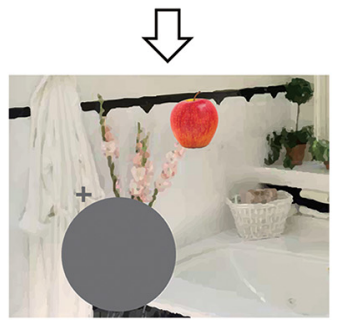

Target reposition

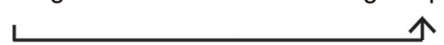
$\uparrow$

Figure 1. PRL training protocol. $\boldsymbol{A}$, Simulated central vision loss with a prescribed PRL, illustrated in a visual field map. The scotoma is a circular gray patch with a radius of $5^{\circ}$. A cross $\left(6.5^{\circ}\right.$ eccentricity, $30^{\circ}$ counterclockwise from the vertical meridian) marks fixation was reached for $2 \mathrm{~s}$, the target jumped to a new location. After six such jumps and stable fixations, subjects reported whether or not the target was present among multiple objects by a key press. Each block ( $\sim 25 \mathrm{~min}$ ) consisted of 40 trials. Subjects completed $40-46$ blocks over 3-4 weeks.

asked to maintain central fixation while to detect a dimming of the target. All the stimuli and the simulated central scotoma were the same as those used in the PRL training experiment. Subjects underwent training for 44 blocks (40 trials per block) over $22 \mathrm{~d}$.

Data analysis. Eye movement data were processed and analyzed using the same method as described in the study by Kwon et al. (2013). Briefly, a parsing algorithm (Gitelman, 2002) was applied to the preprocessed gaze position data to classify saccades and fixations while excluding microsaccades. For each block and each subject, fixation density maps were derived from the retinal positions of the target objects during periods of fixation via kernel density estimation with a bivariate Gaussian kernel (Botev et al., 2010). The PRL was defined as the peak of the density distribution. From the density maps, we calculated a bivariate contour ellipse area (BCEA) that encompassed $68 \%$ of positions around the mean (Castet and Crossland, 2012). In addition to the area of the ellipse, the variance was depicted by the following three parameters: diameter in the major axis, diameter in the minor axis, and angle of the major axis (relative to the radial axis connecting the PRL and the fovea) of the ellipse. The density maps for the first saccade landing site were obtained in a similar manner from the retinal position of the target object at the end point of the first saccade after each target reposition.

\section{Psychophysical measurement of crowding}

Stimuli and design. Visual stimuli consisting of three letter "T"s were presented in the upper left, with the middle letter at $6.5^{\circ}$ eccentricity, $30^{\circ}$ counterclockwise from the vertical meridian. Each letter subtended $0.75^{\circ}$ in the visual field and was presented at $100 \%$ contrast. Background luminance of the display was $25.7 \mathrm{~cd} / \mathrm{m}^{2}$. Two flankers were positioned in either the radial or the tangential direction with respect to the fovea (see Fig. $3 A$ ). In a trial, the target and flankers were presented for $150 \mathrm{~ms}$. After a $500 \mathrm{~ms}$ blank interval, four letter "T"s were presented with different rotating angles. Subjects were asked to identify the target orientation by a mouse click. A correct response was followed by a tone. The next trial began $1 \mathrm{~s}$ after the response. Four blocks were completed for each targetflanker configuration in a counterbalanced order. Each block consisted of 30 trials.

Measuring critical spacing. The crowding effect was quantified by critical spacing, the minimum center-to-center distance between the target and flankers that avoids crowding. For each subject, the critical spacing 
was measured with a psi-marginal adaptive method (Kontsevich and Tyler, 1999) at each target-flanker configuration. The threshold, slope, and lapse rate of a log-Weibull psychometric function were estimated to maximize the posterior probability by the observer's response in each trial. Thresholds were calculated at $63 \%$ correct of a normalized logWeibull function. The critical spacing, which reflects the distance at which identification performance started to drop, was determined by the intersection between slope and a high asymptote. Slope of the psychometric function was determined at the point of inflection at the threshold. The high asymptote was estimated by the weighted average of lapse rate from the psi-marginal method and finger errors from the target-only trials. There were four candidate targets, resulting in a $25 \%$ probability of generating a correct response by random guess.

\section{fMRI measurement of crowding}

Stimuli and design. Similar to the psychophysical test, the fMRI crowding effect was measured with two target-flanker configurations (see Fig. 3B). The stimuli were either three "T"s (target and flankers) or two "T"s (flankers only). Each letter was displayed at a rotation angle randomly chosen from $0^{\circ}, 45^{\circ}, 90^{\circ}$, and $135^{\circ}$ with respect to the configuration axis. To induce a crowding effect in the scanner, the target-flanker center-tocenter distance was fixed at $1.5^{\circ}$, smaller than the average critical spacing across subjects. The centroid of the stimuli was at $6.5^{\circ}$ eccentricity, $30^{\circ}$ counterclockwise from the vertical meridian. The contrast and size (in terms of visual angle) of the letters, and the luminance of the background were identical to the stimuli in the psychophysical tests. The conditions ( 2 axis $\times 2$ target presence) were displayed in a counterbalanced order in eight block-designed runs. Each block consisted of eight trials. During each trial, the letters were alternating between black and white every 50 $\mathrm{ms}$ for two cycles to prevent perceptual fading and to effectively drive the BOLD response. In each trial, the central fixation changed its color (red, yellow, green, or blue) every $50 \mathrm{~ms}$. Subjects were asked to detect whether the last color was the same as either of the first two colors.

fMRI data collection. MRI data were collected using a $3 \mathrm{~T}$ Siemens Prisma scanner with a 32-channel phased-array coil. In the scanner, the stimuli were back projected via a video projector (refresh rate, $60 \mathrm{~Hz}$; spatial resolution, $1024 \times 768$ ) onto a translucent screen placed inside the scanner bore. Subjects viewed the stimuli through a mirror located above their eyes. The viewing distance was $83 \mathrm{~cm}$. BOLD signals were measured at a resolution of $3 \times 3 \times 3 \mathrm{~mm}^{3}$ with a gradient echoplanar imaging sequence (TE, $35 \mathrm{~ms}$; TR, $1 \mathrm{~s}$; FOV, $192 \times 192 \mathrm{~mm}^{2}$; matrix, $64 \times 64$; flip angle, $63^{\circ}$; slice thickness, $3 \mathrm{~mm}$; gap, $0 \mathrm{~mm}$; number of slices, 42 ; slice orientation, axial). A high-resolution 3D structural dataset (MPRAGE; $0.8 \times 0.8 \times 0.8 \mathrm{~mm}^{3}$ resolution) was also collected.

fMRI data preprocessing. MRI data analyses were performed using Freesurfer (version 5.3; http://surfer.nmr.mgh.harvard.edu/) and FSL (version 4.1; FMRIB Software Library; www.fmrib.ox.ac.uk/fsl). The anatomical volume was processed using Freesurfer to reconstruct an inflated cortical surface for each subject. The functional volumes were preprocessed using FSL, including motion correction and high-pass temporal filtering. All functional volumes were registered into 3D space using the subjects' individual high-resolution anatomical images.

Defining regions of interest. Retinotopic visual areas (V1, V2, V3, V3A, $\mathrm{V} 4$, and VO-1/2) were defined by a phase-encoded method (Engel et al., 1994, 1997; Sereno et al., 1995), in which subjects viewed a rotating wedge and an expanding ring that created traveling waves of neural activity in visual cortex. Independent block-design runs were repeated four times to localize regions of interest (ROIs) in V1-V4, VO-1/2, and intraparietal sulcus (IPS). The average number of voxels and the SEMs across subjects were $45 \pm 4$ in V1, $56 \pm 7$ in V2, $52 \pm 10$ in V3, $73 \pm 10$ in V3A, $64 \pm 17$ in V4, $28 \pm 9$ in VO-1/2, and $42 \pm 6$ in IPS. In each block, a target letter with four flankers in both the radial and tangential configurations were presented simultaneously. Each run consisted of eight $16 \mathrm{~s}$ blocks alternating between stimuli presented at the PRL location and its mirrorsymmetric location across the vertical meridian. A general linear model (GLM) with a double-gamma hemodynamic response function was used to identify the ROIs. The ROIs were defined as the voxels that responded stronger to stimuli in the contralateral visual field than in the ipsilateral visual field $(p<0.005)$.
Data analysis. BOLD responses ( $\beta$ values) were estimated with a GLM in each run and averaged across runs for each ROI and each condition. To isolate the crowding-related response and to distinguish the learning effect from general practice effects or common sources of variance (e.g., day-to-day measurement variation, stimulus repetition), we defined the crowding index by subtracting the $\beta$ value in the flanker-only condition from that in the target-plus-flanker condition [crowding index $=\beta$ (target-plus-flanker) $-\beta$ (flanker-only)] and further contrasting the indices between pretest (Pre) and post-test (Post) $[\triangle$ crowding index $=$ crowding index (Post) - crowding index (Pre)].

\section{Experimental design and statistical analyses}

To evaluate the PRL training effect, the variance of fixation/saccadelanding position was compared between the first and the last training blocks using paired-sample $t$ test. To evaluate changes in the crowding effect, the critical spacing was compared between pretests and post-tests using paired-sample $t$ test. In fMRI measurement, the crowding index in each ROI was submitted to repeated-measures ANOVA, with configuration (radial/tangential) and test (pre/post) as two within-subject factors. A post hoc one-sample $t$ test was further conducted on $\triangle$ Crowding index to assess whether the change in the crowding effect was significantly different from zero. The $p$ values were FDR (false discovery rate) corrected for multiple comparisons.

\section{Results}

\section{PRL training}

Subjects' central visual field was occluded by a gray patch using a gaze-contingent display (Fig. 1A). During training, subjects learned to use a prescribed PRL to fixate at a randomly positioned object in a cluttered scene. Each trial ended with a search task to encourage visual processing of the target during object tracking (Fig. $1 B$ ). The average search performance was maintained at $>82 \%$ correct after the third training block. To evaluate the PRL development, eye position data during object tracking were depicted in the following two types of probability density maps: (1) a fixation map, which shows the probability distribution of the retinal position of the target during the fixation period; and (2) a first-saccade landing map, which shows the probability distribution of the retinal position of the target at the completion of the first saccade following each target movement (Fig. 2).

For each type of map, the variance of the retinal position of the target was plotted as a function of training day. Over the training course, the variance decreased, as subjects gradually learned to place the target at the PRL location (Fig. $3 E, F$ ). For the fixation map, compared with the first block, the deviation at the end of the training was reduced by $90.1 \pm 4.3 \%$ and $81.0 \pm 7.8 \%$, respectively, along the major and the minor axis (both $t_{(8)}>8.50, p<$ 0.001 ; Fig. $3 A, C)$. For the first-saccade landing map, the deviation at the end of the training was reduced by $68.9 \pm 11.5 \%$ and $69.7 \pm 10.8 \%$, respectively, along the major and the minor axis (both $t_{(8)}>6.08, p<0.001$; Fig. $3 B, D$ ).

\section{Crowding measurement: psychophysics}

Before and after training, we tested subjects' ability to identify objects in clutter by measuring the effect of crowding. Subjects were asked to report the orientation of a target letter in the middle of two flanking letters (Fig. 4A). The effect of crowding was defined as critical spacing: the minimum distance between the target and flankers that yields reliable target identification performance. Crowding was measured at the PRL location at two configurations along the radial and the tangential axes (Fig. 4B). We compared the behavioral effect of crowding before and after training (Fig. 4C).

We found that the critical spacing was reduced along the radial axis (Fig. $4 D ; t_{(8)}=2.44, p=0.04$ ), but not along the tan- 
A
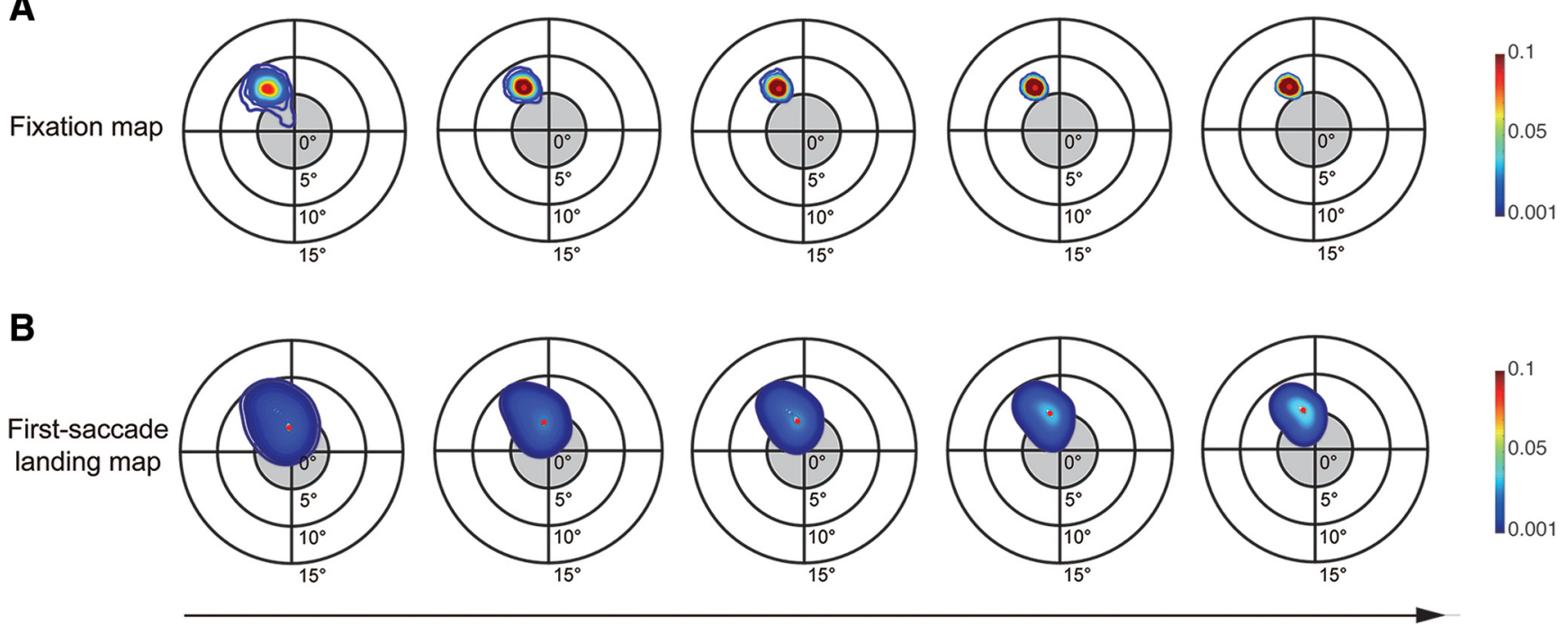

Day 1-5

Day 6-10

Day 11-15

Day 16-20

Day 21-23

Figure 2. Fixation map and first-saccade landing map over the PRL training course. A, Fixation map showing the probability distribution of the retinal position of the target during fixation. $\boldsymbol{B}$, First-saccade landing map showing the probability distribution of the retinal position of the target at the completion of the first saccade following each target movement. Red dot indicates the location of peak density. Gray patch indicates the area of the central scotoma. Maps demonstrate averaged results across subjects and specified training days.

A
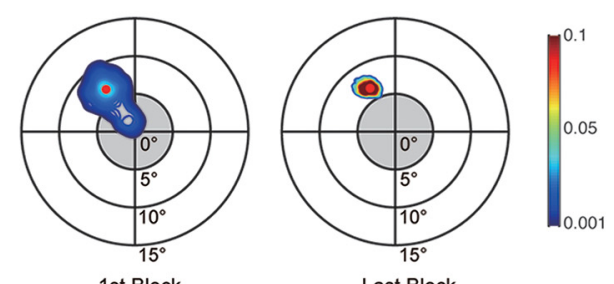

Last Block

B

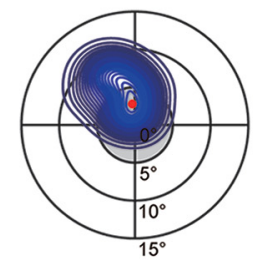

1st Block

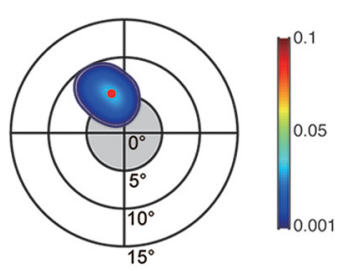

Last Block
C
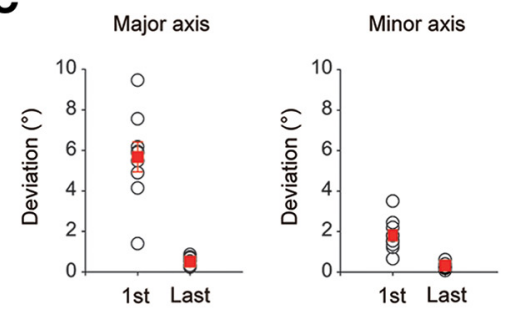

D
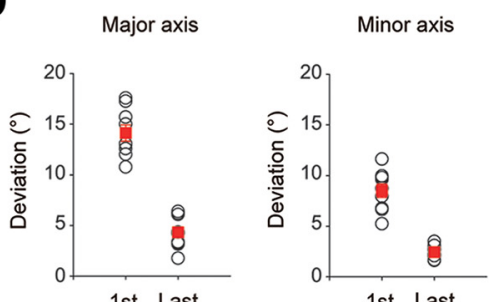

E

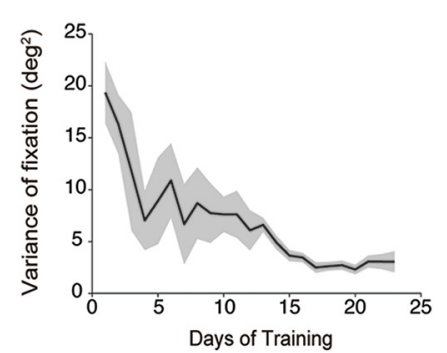

F

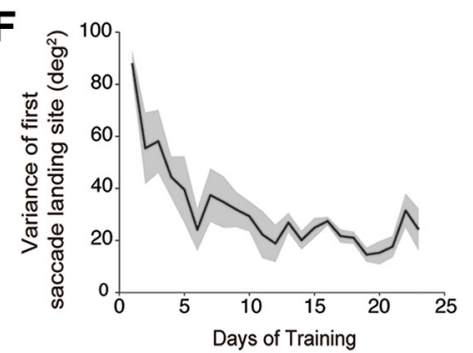

Figure 3. PRL training results. $\boldsymbol{A}$, Fixation map showing the probability distribution of the retinal position of the target during fixation. $\boldsymbol{B}$, First-saccade landing map showing the probability distribution of the retinal position of the target at the completion of the first saccade following each target movement. Red dot indicates the location of peak density. Gray patch indicates the area of the central scotoma. C, Deviation of fixation position in the first and last training block. D, Deviation of first-saccade landing position in the first and last training block. The deviation was computed from a BCEA that encompasses $68 \%$ of positions around the mean. Left column, Diameter along the major axis of the ellipse; right column, diameter along the minor axis of the ellipse. The black dot depicts single-subject data. The red square and bars depict the mean \pm SEM across subjects. $\boldsymbol{E}$, Learning curve for the fixation map. $\boldsymbol{F}$, Learning curve for the first-saccade landing map. Variance of the retinal position of the target, defined as the BCEA area, is plotted as a function of training day. The shaded area denotes \pm 1 SEM across subjects.

gential axis $\left(t_{(8)}=0.38, p=0.71\right)$. To explore the relationship between the PRL training and the observed changes in the crowding effect, we calculated the correlation coefficient between the improvement in the eye movement control and the change in crowding effect across subjects (Fig. $4 E$ ). We found a significant correlation between the reduction in the deviation of the firstsaccade landing site along the major axis and the reduction of crowding effect in the radial configuration $(r=0.68, p=0.045)$.

To test whether the changes in the crowding effect resulted from a potential change of attention, another eight subjects underwent training over a comparable length of time. The training did not involve any oculomotor task. Instead, subjects were asked to deploy covert attention to a target at the PRL location. After training, the critical spacing was reduced by $1.12 \pm 0.21^{\circ}$ along the radial axis and $1.08 \pm 0.25^{\circ}$ along the tangential axis. The reduction was significant along both axes (both $t_{(7)}>4.00, p<$ $0.005)$, different from the radial-specific reduction induced by PRL training. These results suggest that an attention account is unlikely to explain the change in the crowding effect after PRL induction training.

We also tested the influence on the crowding effect from the test-retest familiarity. Nine naive subjects underwent the behavioral crowding tests without any training. The critical spacing did not differ between pretests and post-tests in both the radial and 
A

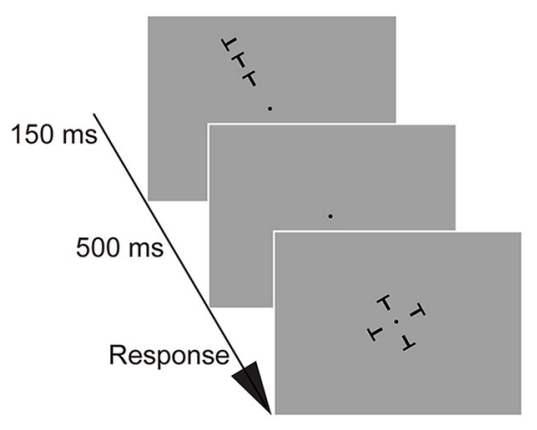

B

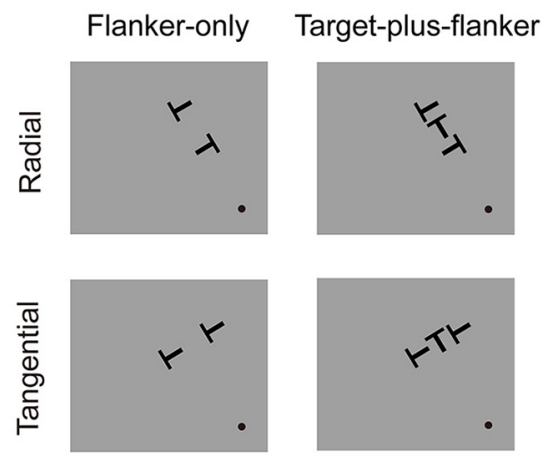

C

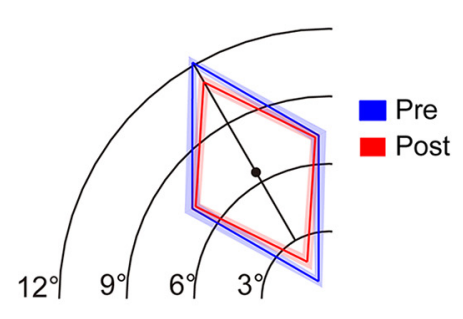

D

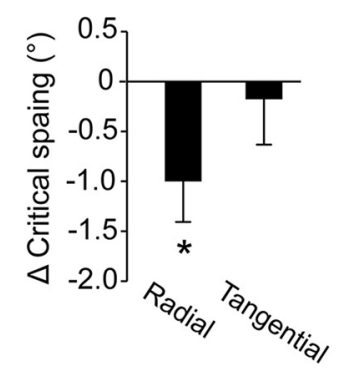

E

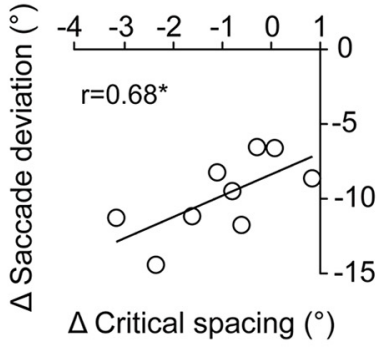

Figure 4. Protocols and behavioral results of crowding measurements. $A$, Stimuli and task in the behavioral test. Subjects were asked to report the orientation of a target " $T$ " in the middle of two flankers in a four-alternative forced-choice (4-AFC) trial in an adaptive staircase measurement. $\boldsymbol{B}$, Stimuli configuration in the fMRI measurement of crowding. Flankers were displayed in radial or tangential configuration. C, Crowding zone plotted in the visual field map based on the critical spacing measured in Pre and Post tests. $\boldsymbol{D}$, Changes in the critical spacing along the radial and tangential axes. $\boldsymbol{E}$, Interindividual correlation between the change in first-saccade landing deviation along the major axis and the change in the critical spacing along the radial axis. Error bars denote \pm 1 SEM across subjects. Asterisks indicate significance: ${ }^{*} p<0.05$.

the tangential configurations (both $t_{(8)}<0.78, p>0.46$ ). These results suggest that PRL training is needed to induce the reduction in the crowding effect.

\section{Crowding measurement: fMRI}

Only subjects from the PRL training group participated in the fMRI experiment. Using fMRI, we probed the neural substrates underlying the behavioral changes of crowding in areas V1-V4, VO-1/2, and IPS (Fig. 5A). For each stimulus configuration, crowding-related signals were assessed by contrasting BOLD signals between target-plus-flanker and flanker-only conditions (Fig. 5B). A repeated-measures ANOVA with configuration (radial/tangential) and test (Pre/Post) as two factors revealed a significant interaction effect in V1 $\left(F_{(1,8)}=7.16, p=0.034\right)$, V2 $\left(F_{(1,8)}=6.43, p=0.035\right)$, $\mathrm{V} 3\left(F_{(1,8)}=9.70, p=0.026\right), \mathrm{V} 3 \mathrm{~A}\left(F_{(1,8)}=20.62, p=0.014\right), \mathrm{V} 4$ $\left(F_{(1,8)}=7.68, p=0.034\right)$, VO-1/2 $\left(F_{(1,8)}=9.59, p=0.026\right)$, and IPS $\left(F_{(1,8)}=9.73, p=0.026\right)$. The $p$ values were FDR corrected.

We quantified the training-induced change by contrasting the crowding index between pretest and post-test (Fig. 5C). A positive value indicates a weaker crowding effect after training, while a negative value indicates a stronger crowding effect after training. Across these visual areas, training led to a radial-specific reduction of the crowding effect. A significant reduction of the crowding effect was observed in all of the ROIs in the radial configuration (all $t_{(8)}>3.26, p<0.014$, FDR corrected), but not in the tangential configuration (all $t_{(8)}<1.12, p>0.30$, FDR corrected). We calculated correlation coefficients between the reduction in the behavioral crowding effect and the change in fMRI crowding index in the radial condition. A significant correlation was observed in VO- $1 / 2(r=0.71, p=0.032$, uncor- rected). No significant correlation was observed in other ROIs (all $r<0.39, p>0.29$, uncorrected).

\section{Discussion}

Our study provides the following psychophysical and neuroimaging findings: (1) training with simulated central vision loss induced a PRL in normally sighted adults; (2) PRL training reduced crowding along the radial direction; and (3) training reduced crowding-related suppression in the fMRI BOLD response across visual areas $\mathrm{V} 1-\mathrm{V} 4, \mathrm{VO}-1 / 2$, and IPS. These perceptual and neural changes suggest that developing a PRL reshapes the oculomotor control and sensory coding in peripheral vision.

One or several PRLs often develop in people with central vision loss over a long-term period (Cummings et al., 1985; Crossland et al., 2005), but the nature and dynamics of this adjustment remain unclear. By inducing a PRL in normally sighted adults, we avoided several confounds in clinical studies (e.g., the variability in the size, inhomogeneity, and the continuous degenerative progress). To ensure that the premeasurements and postmeasurements were comparable, all PRLs were induced at a fixed location. The PRL was set at $1.5^{\circ}$ away from the border of the simulated central scotoma, as patients tend to develop their PRL close to the impaired retinal area (Fletcher and Schuchard, 1997). The observed improvements in the fixation stability and the saccadic precision were consistent with the oculomotor adaptability previously reported in monkeys with bilateral foveal retinal lesions (Heinen and Skavenski, 1992). A notable difference, however, is that the scotoma in the current study was not able to induce a perceptual filling-in effect (Ramachandran and Greg- 
A

V1

V2

V3

V3A

V4

VO-1/2

IPS
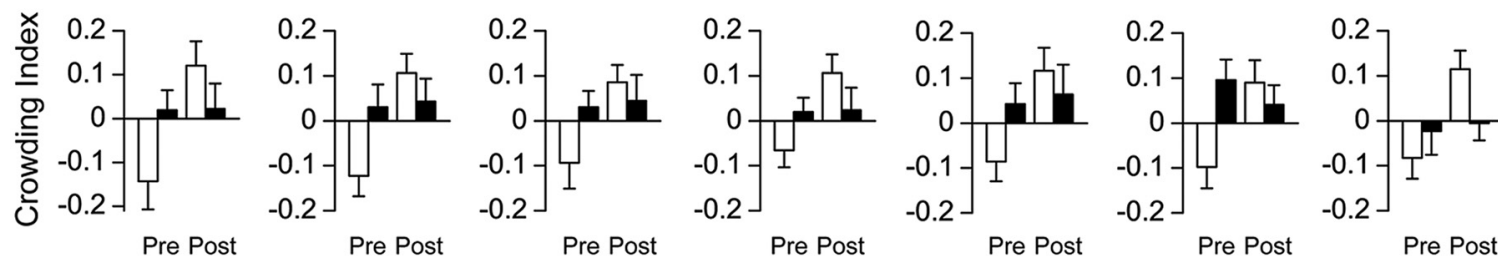

$\square$ Radial

- Tangential

B

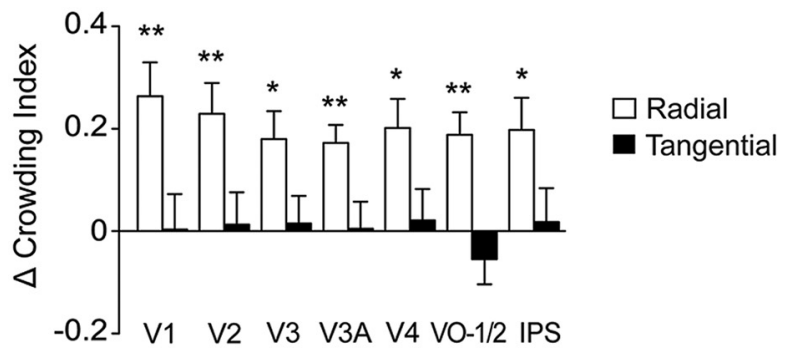

C

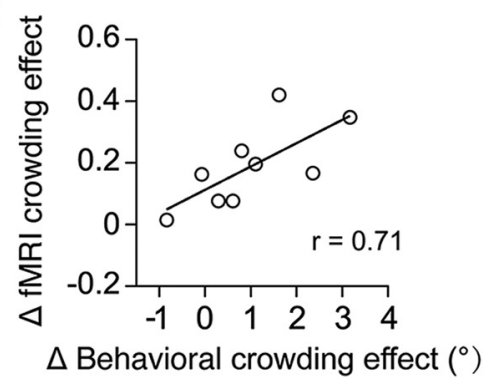

Figure 5. fMRI results of crowding measurements. $\boldsymbol{A}$, fMRI crowding effect before and after PRL training: Crowding index $=\beta$ (target-plus-flanker) $-\beta$ (flanker-only). $\boldsymbol{B}$, Change of fMRI crowding effect: $\triangle$ Crowding index $=$ Crowding index(Post) - Crowding index(Pre). $\boldsymbol{C}$, Interindividual correlation between the changes in behavioral and fMRI measurements of crowding. $\triangle$ Behavioral crowding effect, Reduction in the critical spacing along the radial axis; $\triangle \mathrm{fMRI}$ crowding effect, increase in crowding index in radial condition in V0-1/2. Error bars denote $\pm 1 \mathrm{SEM}$ across subjects. Asterisks indicate significance: ${ }^{*} p<0.05,{ }^{* *} p<0.01$, FDR corrected.

ory, 1991; Zur and Ullman, 2003), which is often experienced by patients, possibly due to its visible boundary. Kwon et al. (2013) tested the transfer effect of PRL induction in an invisible scotoma condition by matching the color and the luminance of the scotoma to the background. Kwon et al. (2013) found that the PRL induction effect can be generalized independent of the scotoma visibility.

Importantly, we found that the development of a PRL affects the spatial extent of crowding. A link between the oculomotor control and form processing in peripheral vision has been suggested in previous psychophysical studies. By measuring the size of the crowding zone and the saccade-landing zone, Greenwood et al. (2017) demonstrated that the saccadic precision and crowding covary across the visual fields. Harrison et al. (2013) showed that eye movement preparation effectively reduces the critical spacing of the crowding zone. Consistently, we observed a correlation between the change in the saccadic precision and the change in the crowding effect. The reduction in the variance of the first-saccade landing site correlated with the reduction of critical spacing along the radial direction across subjects. These findings indicate that despite the general dissociation between the perception and saccadic eye movement (Tavassoli and Ringach, 2010; Lisi and Cavanagh, 2015), saccadic eye movement may influence the perceptual process over time.

A possible mechanism underlying the relationship between saccades and crowding has been described by Nandy and Tjan (2012). Their model proposes that when saccades bring an object of interest into the fovea, the visual image moves along the radial direction across the retina. This provides distorted visual input, leading to inappropriate contextual interactions. The model predicts that, when saccades are redirected to a PRL, less retinal motion occurs along the radial direction with respect to the fovea. Over the course of training, the visual system adapts to a new set of image statistics that contains less overintegration of information along the radial axis. Consequently, the features of flankers positioned radial to the target with respect to the fovea become less bound with the target, leading to a reduction of the crowding effect along the radial direction. In addition to the empirical findings from the present study, a reduction in the anisotropy of the crowding zone has been observed in macular degeneration patients with long-term central vision loss at their established PRL (Chung, 2013). The model also predicts reorganization of the crowding zones at other spatial locations according to the new visual reference locus. Our ongoing work aims to further test these predictions.

Parallel to the shrinkage of the crowding zone, we observed a specific reduction in the crowding effect in the visual cortex. To overcome the difficulty in separating the signals from closely spaced targets and flankers, we defined the crowdingrelated response by contrasting the BOLD responses between the target-plus-flanker and flanker-only conditions (Chen et al., 2014; Kwon et al., 2014; Millin et al., 2014). Our crowding index reflects a combination of signal increase from the presence of a target and signal decrease from the suppressive interaction between the target and the flankers. When the crowding-related suppression was reduced, the signal increase evoked by the target became dominant. As a result, the sign of the crowding index flipped from negative to positive in the radial condition after training.

Changes in crowding-related cortical suppression were observed with attention directed away from the stimuli. It has been shown that attention modulates crowding-related cortical suppression at a very early stage of visual processing (Chen et al., 2014), and that attention alters the neural representation of the crowded feature along the visual hierarchy (Chen et al., 2018). To minimize the influence of attention on the BOLD signals evoked by the peripheral stimuli, we asked subjects to perform a rapid color detection task at the central fixation during the scan. Such a protocol has been used to reflect a bottom-up stimulus-driven component of crowding, which starts as early as V1 (Kwon et al., 2014; Millin et al., 2014). In addition to V1, previous neurophysiological and brain-imaging studies have suggested that crowding 
could also take place in V2 (Fang and He, 2008), V3 (Bi et al., 2009), and V4 or higher-level visual areas (Motter, 2006; Liu et al., 2009; Anderson et al., 2012). Consistent with the idea that crowding originates in the early visual cortex, with its perceptual consequence influenced by later visual area (Anderson et al., 2012; Chen et al., 2018), we observed a radial-specific reduction across the visual areas and a significant correlation between changes in the behavioral and fMRI crowding effect in VO-1/2.

The observed anisotropic change in the crowding effect might reflect a rewiring in the lateral connections in the visual cortex. It is known that the cortex reorganizes its effective local connections and responses following peripheral or central alterations of inputs (Buonomano and Merzenich, 1998). While reorganization in the cortical response, manifested as topological remapping, has been examined in many fMRI studies (Masuda et al., 2008; Dilks et al., 2009; Liu et al., 2010; Baseler et al., 2011), reorganization in the local connections, manifested as changes in the interaction between adjacent stimuli, has been little studied. The current study tested this connection-based reorganization hypothesis inspired by the saccade-confounded image statistic model proposed by Nandy and Tjan (2012). As the PRL becomes a pseudofovea, connections that bind features from the clutter surrounding the target could be reshaped. Consistent with this hypothesis, we observed changes in the crowding-related cortical suppression and the critical spacing of the crowding zone. This form of reorganization alleviates the fundamental limit of peripheral vision to compensate for central vision loss.

Training improves performance for many visual tasks, a phenomenon referred to as visual perceptual learning (Fahle and Poggio, 2002; Watanabe and Sasaki, 2015). It should be noted that our training protocol is different from typical perceptual learning studies. Previous psychophysical studies have shown that training enhances the ability to identify or detect the target in the midst of flankers (Sun et al., 2010; Hussain et al., 2012; Mayhew et al., 2012; Zhu et al., 2016; Maniglia et al., 2018). In these studies, the same task was used in both the training and test phases, and the learning effect was often specific to features of the trained stimulus. In the present study, subjects were trained to track objects in cluttered backgrounds, while being tested in an orientation identification task with letter stimuli. Our findings shed light on developing effective rehabilitation protocols that transfer traininginduced improvement to everyday tasks.

\section{References}

Aguilar C, Castet E (2011) Gaze-contingent simulation of retinopathy: some potential pitfalls and remedies. Vision Res 51:997-1012.

Anderson EJ, Dakin SC, Schwarzkopf DS, Rees G, Greenwood JA (2012) The neural correlates of crowding-induced changes in appearance. Curr Biol 22:1199-1206.

Baseler HA, Gouws A, Haak KV, Racey C, Crossland MD, Tufail A, Rubin GS, Cornelissen FW, Morland AB (2011) Large-scale remapping of visual cortex is absent in adult humans with macular degeneration. Nat Neurosci 14:649-655.

Bi T, Cai P, Zhou T, Fang F (2009) The effect of crowding on orientationselective adaptation in human early visual cortex. J Vis 9(11):13, 1-10.

Botev ZI, Grotowski JF, Kroese DP (2010) Kernel density estimation via diffusion. Ann Statist 38:2916-2957.

Bouma H (1970) Interaction effects in parafoveal letter recognition. Nature 226:177-178.

Buonomano DV, Merzenich MM (1998) Cortical plasticity: from synapses to maps. Annu Rev Neurosci 21:149-186.

Castet E, Crossland M (2012) Quantifying eye stability during a fixation task: a review of definitions and methods. Seeing Perceiving 25:449-469.
Chen J, He Y, Zhu Z, Zhou T, Peng Y, Zhang X, Fang F (2014) Attentiondependent early cortical suppression contributes to crowding. J Neurosci 34:10465-10474.

Chen N, Bao P, Tjan BS (2018) Contextual-dependent attention effect on crowded orientation signals in human visual cortex. J Neurosci 38: $8433-8440$

Chung ST (2013) Cortical reorganization after long-term adaptation to retinal lesions in humans. J Neurosci 33:18080-18086.

Crossland MD, Culham LE, Kabanarou SA, Rubin GS (2005) Preferred retinal locus development in patients with macular disease. Ophthalmology 112:1579-1585.

Crossland MD, Engel SA, Legge GE (2011) The preferred retinal locus in macular disease: toward a consensus definition. Retina 31:2109-2114.

Cummings RW, Whittaker SG, Watson GR, Budd JM (1985) Scanning characters and reading with a central scotoma. Am J Optom Physiol Opt 62:833-843.

Dilks DD, Baker CI, Peli E, Kanwisher N (2009) Reorganization of visual processing in macular degeneration is not specific to the "preferred retinal locus". J Neurosci 29:2768-2773.

Engel SA, Rumelhart DE, Wandell BA, Lee AT, Glover GH, Chichilnisky EJ, Shadlen MN (1994) fMRI of human visual cortex. Nature 369:525.

Engel SA, Glover GH, Wandell BA (1997) Retinotopic organization in human visual cortex and the spatial precision of functional MRI. Cereb Cortex 7:181-192.

Fahle M, Poggio T (2002) Perceptual learning. Cambridge, MA: MIT.

Fang F, He S (2008) Crowding alters the spatial distribution of attention modulation in human primary visual cortex. J Vis 8(9):6, 1-9.

Fletcher DC, Schuchard RA (1997) Preferred retinal loci relationship to macular scotomas in a low-vision population. Ophthalmology 104:632638.

Gitelman DR (2002) ILAB: a program for postexperimental eye movement analysis. Behav Res Methods Instrum Comput 34:605-612.

Greenwood JA, Szinte M, Sayim B, Cavanagh P (2017) Variations in crowding, saccadic precision, and spatial localization reveal the shared topology of spatial vision. Proc Natl Acad Sci U S A 114:E3573-E3582.

Harrison WJ, Mattingley JB, Remington RW (2013) Eye movement targets are released from visual crowding. J Neurosci 33:2927-2933.

Heinen SJ, Skavenski AA (1992) Adaptation of saccades and fixation to bilateral foveal lesions in adult monkey. Vision Res 32:365-373.

Hussain Z, Webb BS, Astle AT, McGraw PV (2012) Perceptual learning reduces crowding in amblyopia and in the normal periphery. J Neurosci 32:474-480.

Kontsevich LL, Tyler CW (1999) Bayesian adaptive estimation of psychometric slope and threshold. Vision Res 39:2729-2737.

Kwon M, Nandy AS, Tjan BS (2013) Rapid and persistent adaptability of human oculomotor control in response to simulated central vision loss. Curr Biol 23:1663-1669.

Kwon M, Bao P, Millin R, Tjan BS (2014) Radial-tangential anisotropy of crowding in the early visual areas. J Neurophysiol 112:2413-2422.

Lisi M, Cavanagh P (2015) Dissociation between the perceptual and saccadic localization of moving objects. Curr Biol 25:2535-2540.

Liu T, Jiang Y, Sun X, He S (2009) Reduction of the crowding effect in spatially adjacent but cortically remote visual stimuli. Curr Biol 19:127132.

Liu T, Cheung SH, Schuchard RA, Glielmi CB, Hu X, He S, Legge GE (2010) Incomplete cortical reorganization in macular degeneration. Invest Ophthalmol Vis Sci 51:6826-6834.

Luo G, Satgunam P, Peli E (2012) Visual search performance of patients with vision impairment: effect of JPEG image enhancement. Ophthalmic Physiol Opt 32:421-428.

Maniglia M, Soler V, Cottereau B, Trotter Y (2018) Spontaneous and training-induced cortical plasticity in MD patients: hints from lateral masking. Sci Rep 8:90.

Masuda Y, Dumoulin SO, Nakadomari S, Wandell BA (2008) V1 projection zone signals in human macular degeneration depend on task, not stimulus. Cereb Cortex 18:2483-2493.

Mayhew SD, Li S, Kourtzi Z (2012) Learning acts on distinct processes for visual form perception in the human brain. J Neurosci 32:775-786.

Millin R, Arman AC, Chung ST, Tjan BS (2014) Visual crowding in V1. Cereb Cortex 24:3107-3115. 
Motter BC (2006) Modulation of transient and sustained response components of $\mathrm{V} 4$ neurons by temporal crowding in flashed stimulus sequences. J Neurosci 26:9683-9694.

Nandy AS, Tjan BS (2012) Saccade-confounded image statistics explain visual crowding. Nat Neurosci 15:463-469, S1-S2.

Ramachandran VS, Gregory RL (1991) Perceptual filling in of artificially induced scotomas in human vision. Nature 350:699-702.

Sereno MI, Dale AM, Reppas JB, Kwong KK, Belliveau JW, Brady TJ, Rosen BR, Tootell RB (1995) Borders of multiple visual areas in humans revealed by functional magnetic resonance imaging. Science 268:889-893.

Sun GJ, Chung STL, Tjan BS (2010) Ideal observer analysis of crowding and the reduction of crowding through learning. J Vis 10(5):16, 1-14.
Tavassoli A, Ringach DL (2010) When your eyes see more than you do. Curr Biol 20:R93-R94.

Toet A, Levi DM (1992) The two-dimensional shape of spatial interaction zones in the parafovea. Vision Res 32:1349-1357.

Watanabe T, Sasaki Y (2015) Perceptual learning: toward a comprehensive theory. Annu Rev Psychol 66:197-221.

Whitney D, Levi DM (2011) Visual crowding: a fundamental limit on conscious perception and object recognition. Trends Cogn Sci 15:160-168.

Zhu Z, Fan Z, Fang F (2016) Two-stage perceptual learning to break visual crowding. J Vis 16(6):16, 1-12.

Zur D, Ullman S (2003) Filling-in of retinal scotomas. Vision Res 43:971982. 